\section{Hemodynamic ischemia of the anterior choroidal artery and reversal by carotid artery stenting 10 years after ipsilateral superficial temporal artery-middle cerebral artery bypass for symptomatic left middle cerebral artery stenosis}

\author{
Hayato Suzuki,1,2 Suguru Yamaguchi,1 \\ Katsuhiro Nishino, 1 Taizen Nakase, 2 \\ Hiroaki Shimizu ${ }^{2}$
}

1Department of Neurosurgery, Kakunodate General Hospital, Akita; ${ }^{2}$ Department of Neurosurgery, Akita University Graduate School of Medicine, Akita, Japan

\begin{abstract}
The pathogenesis of anterior choroidal artery (AChA) territory infarction includes various mechanisms, but hemodynamic causes are rare and difficult to diagnose. 77year-old man, who had moderate left ICA stenosis and he had treated with STA-MCA bypass surgery for severe symptomatic left MCA stenosis 10 years earlier, was admitted with right hemiparesis and confused state. On admission, magnetic resonance imaging and angiography demonstrated patent bypass, but severe stenosis of left ICA with no opacification of the left AChA and A1 portion of the left ACA. Diffusionweighted imaging demonstrated ischemic lesion in the left corona radiata. Together with clinical findings, hemodynamic ischemia of the AChA region was suspected and left carotid artery stenting resulted in prompt improvement of symptoms. Hemodynamic ischemia of the AChA territory is rare, however, should be considered as a potential target of treatment when the ipsilateral ICA, A1 and M1 show stenoocclusive lesions
\end{abstract}

\section{Introduction}

Anterior choroidal artery (AChA) infarction is classically known to be Abbie syndrome, presenting triad of hemiparesis, hemianesthesia, and hemianopia. ${ }^{1}$ In these days, more symptoms have been reported in the AChA territory infarction. ${ }^{2}$ Large infarction of AChA territory had worse clinical progression than small infarction of that. ${ }^{3}$
Regarding the etiology, Bruno et al. suggested AChA territory infarction considers to associate with small-vessel disease, not with carotid artery stenosis and cardiac emborism. ${ }^{4}$ A previous study reported the AChA territory infarction was due to small vessel disease in $14 \%$, large vessel disease in 38\%, and cardio-embolism in $4.8 \% .^{5}$

We treated a patient of hemodynamic ischemia in the anterior choroidal artery (AchA) territory due to acute ICA occlusion 10 years after the ipsilateral superficial temporal artery (STA)-middle cerebral artery (MCA) bypass, with carotid artery stenting.

\section{Case Report}

A 77-year-old Japanese male was hospitalized at emergency room with right hemiparesis and confused state. On his medical history, had treated with superficial temporal artery (STA)-middle cerebral artery (MCA) bypass surgery for severe symptomatic left MCA stenosis 10 years earlier. Moreover, cognitive heart failure and post percutaneous coronary intervention for angina were noted. At the time of his admission, his neurological findings were confused state (Glasgow Coma Scale; E1V1M4), dysarthria, and right hemiparesis (manual muscle testing: upper limbs 4/5 lower limbs 3/5).

Diffusion-weighted imaging (DWI) showed a hyperintense area in the left corona radiata (Figure 1A). Magnetic resonance angiography (MRA) showed a hypoplastic posterior communicating artery (PCoA) (Figure 1B). Conventional angiography revealed near-occlusion (99\% stenosis from NASCET criteria) of the left cervical internal carotid artery (Figure 1C), a patent STA-MCA bypass with occlusion of the left M1 (Figure 1D) and severe stenosis of left A1 was visualized via the anterior communicating artery (ACoA) (Figure 1E). Angiography suggested poor perfusion of the AChA territory (Figure 1C-E).

The symptoms and imaging findings suggested hemodynamic ischemia of the AChA territory. After these examinations were over, his neurological findings were confused state was improved (Glasgow Coma Scale; E3V4M5), however, dysarthria, and right hemiparesis (manual muscle testing: upper limbs $4 / 5$ lower limbs $3 / 5$ ) were remained.

Emergent revascularization therapy was considered to prevent recurrent attacks and to avoid progression of AChA territory infarction.

Carotid endarterectomy (CEA) was
Correspondence: Hayato Suzuki, Department of Neurosurgery, Kakunodate General Hospital, 3 Iwase Kakunodate-Machi, Senboku-city, Akita 014-0394, Japan.

Tel.: +81.187.542111; Fax: +81.187.542715. E-mail: haya19870702@gmail.com

Key words: anterior choroidal artery; hemodynamic ischemia; carotid artery stenosis.

Acknowledgements: the authors wish to thank the radiology technicians at the Kakunodade general hospital for their valuable assistance with the surgery in the present case.

Contributions: Surgery was performed by SY and HS; KN, TN and HS supervised the entire work.

Conflict of interest: the authors declare no potential conflict of interests.

Funding: none

Received for publication: 12 September 2018 Accepted for publication: 13 September 2018.

This work is licensed under a Creative Commons Attribution NonCommercial 4.0 License (CC BY-NC 4.0).

(C) Copyright H. Suzuki et al., 2018

Licensee PAGEPress, Italy

Neurology International 2018; 10:7867

doi:10.4081/ni.2018.7867

considered as high-risk treatment because general anesthesia was necessary for operation and longer interception of CCA flow, these induce poor perfusion of left AChA territory and his medical history had cognitive heart failure due to myocardial infarction. We selected emergent CAS as the optimal treatment for the patient, he was receiving dual antiplatelet treatment for left ICA stenosis, and CAS enable shorter CCA occlusion time and under local anesthesia.

Under local anesthesia, a closed cell stent (Carotid Wallstent ${ }^{\mathrm{TM}}$ Monorail ${ }^{\mathrm{TM}}$; Boston Scientific, Marlborough, Massachusetts, United State.) was introduced. Each step was performed under distal blocking. The occlusion time of ICA was 20 minutes.

We therefore performed left carotid artery stenting (CAS), resulting in better antegrade visualization of the left ICA bifurcation (Figure 1F).

Neurological symptoms were not getting worse and postoperative DWI showed no enlargement of the ischemic regions. $\mathrm{He}$ was discharged after rehabilitation under antiplatelet administration with a modified Rankin Scale score of 1. 


\section{Discussion}

The AChA arises from the supraclinoid segment of the ICA as first major branch. ${ }^{6}$ The AChA supplies not only ventricle area, basal ganglia, and motor and visual pathway. ${ }^{7}$ A previous epidemiologic study reported AChA territory infarction was due to small vessel disease in $14 \%$, large vessel disease in $38 \%$, and cardio-embolism in $4.8 \% .^{5}$ In cases with ICA occlusion proximal to the AChA, major collateral flow to the ipsilateral AChA territory may be from the $\mathrm{PCoA}, \mathrm{ACoA}$, and/or retrograde flow from the MCA through a leptomeningeal anastomosis. 8,9 Moreover, the AChA receives collaterals from the posterior choroidal arteries, and although the degree may vary, this circulation is usually insufficient to prevent infarction. 10 Therefore, unless many collaterals supplies are developed at AChA territory, hemodynamic ischemia might be rare. In the present case, contralateral lower extremity-dominant motor weakness, sensory dysfunction, and a confused state were present, these findings

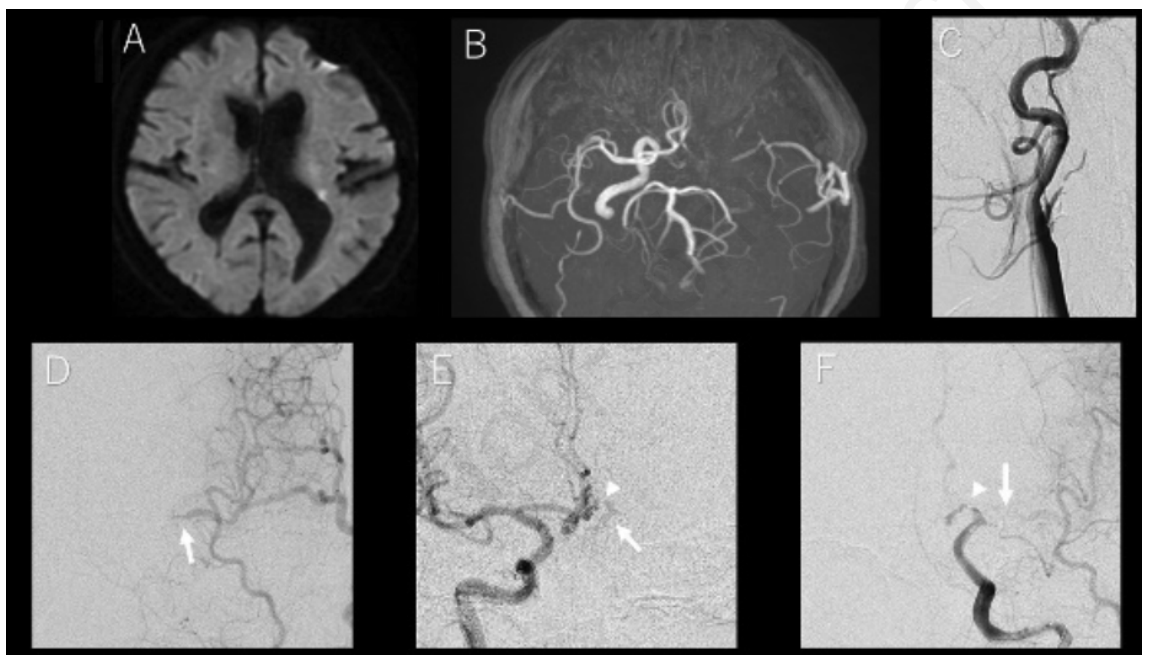

Figure 1. A) Diffusion-weighted imaging on admission shows a spotty, high-intensity lesion in the left corona radiata. B) Magnetic resonance angiography shows a patent superficial temporal artery (STA)-middle cerebral artery (MCA) bypass and MCA occlusion. The left A1 portion of the anterior cerebral artery (ACA) is slightly visualized. The left internal carotid and posterior communicating arteries are not visualized. C) Left common carotid artery (CCA) angiography taken immediately following the MR studies shows near-occlusion of the left internal carotid artery (ICA). D) Antero-posterior (AP)view left external carotid artery (ECA) angiogram shows patency of the STA-MCA bypass and occlusion of the left M1 (white arrow). E) AP-view right ICA angiogram shows slight opacification of the terminal portion of the left ICA (arrow), although the severely stenotic left A1 (arrowhead) segment of the left anterior choroidal artery is not clear. F) APview left CCA angiogram after successful carotid artery stenting shows better visualization of the ICA. Stenosis of the A1 (arrowhead) and occlusion of the M1 (arrow) are evident after branching from the anterior temporal artery.

\section{Conclusions}

A case of hemodynamic ischemia in the anterior choroidal artery (AChA) territory due to ICA occlusion was successfully treated with CAS. Few reports were available on hemodynamic ischemia of AChA territory, so we thought the present case was educational about symptoms and image findings. We should take in account of hemodynamic ischemia of the AChA territory, as a potential target of treatment when the ipsilateral ICA, A1 and M1 show stenoocclusive lesions.

\section{References}

1. Abbie AA. The clinical significance of the anterior choroidal artery. Brain 1933; 56: 233-46.

2. Chausson N, Joux J, Saint-Vil M, et al. Infarction in the anterior choroidal artery territory: clinical progression and prognosis factors. J Stroke Cerebrovasc Dis $2014 ; 23: 2012-7$.

3. Ois A, Cuadrado-Godia E, Solano A, et al. Acute ischemic stroke in anterior choroidal artery territory. J Neurol Sci 2009;281:80-4.

4. Bruno A, Graff-Radford NR, Biller J, Adams HP Jr. Anterior choroidal artery territory infarction: a small vessel dis ease. Stroke 1989;20:616-9.

5. Palomeras E, Fossas P, Cano AT, et al. Anterior choroidal artery infarction: a clinical, etiologic and prognostic study. Acta Neurol Scand 2008;118:42-7.

6. Uz A, Erbil K, Esmer A. The origin and relations of the anterior choroidal artery: an anatomical study. Folia Morphol (Warsz) 2005;64:269-72.

7. Hussein S, Renella RR, Dietz H. Microsurgical anatomy of the anterior choroidal artery. Acta Neurochir (Wien) 1988;92:19-28.

8. Hendrikse J, Hartkamp MJ, Hillen B, et al. Collateral ability of the circle of Willis in patients with unilateral internal carotid artery occlusion: border zone infarcts and clinical symptoms. Stroke 2001;32:2768-73.

9. Miralles M, Dolz JL, Cotillas J, et al. The role of the circle of Willis in carotid occlusion: assessment with phase contrast MR angiography and transcranial duplex. Eur J Vasc Endovasc Surg 1995;10:424-30.

10. Takahashi S, Suga T, Kawata Y, Sakamoto K. Anterior choroidal artery: angiographic analysis of variations and anomalies. AJNR Am J Neuroradiol 1990;11:19-29. 